\title{
Structural Safety Monitoring of High Arch Dam Using Improved ABC-BP Model
}

\author{
Yantao Zhu, ${ }^{1,2,3}$ Chongshi Gu, ${ }^{1,2,3}$ Erfeng Zhao, ${ }^{1,2,3}$ Jintao Song, ${ }^{1,2,3}$ and Zhiyun Guo ${ }^{1,2,3}$ \\ ${ }^{1}$ State Key Laboratory of Hydrology, Water Resources and Hydraulic Engineering, Hohai University, Nanjing 210098, China \\ ${ }^{2}$ National Engineering Research Center of Water Resources Efficient Utilization and Engineering Safety, \\ Hohai University, Nanjing 210098, China \\ ${ }^{3}$ College of Water Conservancy and Hydropower, Hohai University, Nanjing 210098, China
}

Correspondence should be addressed to Yantao Zhu; zyt50@163.com

Received 30 June 2016; Revised 28 August 2016; Accepted 29 August 2016

Academic Editor: Filippo Ubertini

Copyright (C) 2016 Yantao Zhu et al. This is an open access article distributed under the Creative Commons Attribution License, which permits unrestricted use, distribution, and reproduction in any medium, provided the original work is properly cited.

\begin{abstract}
The establishment of a structural safety monitoring model of a dam is necessary for the evaluation of the dam's deformation status. The structural safety monitoring method based on the monitoring data is widely used in traditional research. On the basis of the analysis of the high arch dam's deformation principles, this study proposes a structural safety monitoring method derived from the dam deformation monitoring data. The method first analyzes and establishes the spatial and temporal distribution of high arch dam's safety monitoring, overcoming the standard artificial bee colony (ABC) algorithm's shortcoming of easily falling into the local optimum by adopting the adaptive proportion and average Euclidean distance afterwards. The improved $\mathrm{ABC}$ algorithm is used to optimize the backpropagation (BP) neural network's initial weight and threshold. The application example proves that ABC-BP model's improvement method is important for the establishment of a high arch deformation safety monitoring model and can effectively improve the model's fitting and forecasting ability. This method provides a reference for the establishment of a structural safety monitoring model of dam and provides guidance for the establishment of a forecasting model in other fields.
\end{abstract}

\section{Introduction}

Analyzing the monitoring data and establishing a structural safety monitoring model of dam are necessary to the evaluation of dam deformation status and are important links to carry out structural safety monitoring, instruct construction, and improve the design method. They play important roles in the construction, operation, and other stages of the dam.

Structural safety monitoring of dams began in the 1890s and was first applied in the displacement monitoring of the Bach gravity dam in Germany. Statistical regression method began to be used in the 1950s for quantitative analysis of dams' safety monitoring information. Deterministic and hybrid models of concrete dams were first presented in the 1970s. The models combined finite element theoretical results with actual data to derive the dam's actual physical parameter $[1,2]$. Chen used regression method in 1974 to analyze the monitoring information and explained its physical causes [3]. $\mathrm{Wu}$ derived a dam's time effect displacement expression on the basis of concrete creep theory. They used the periodic function to stimulate temperature and other periodic loads and then used nonlinear least square method for parameter estimation $[4,5]$. They proposed the establishment of the dam crack opening degree statistic model and analysis method, the time series analysis method of horizontal displacement of dam crest, and the multiple-arch dam displacement deterministic model's theory and method. These methods were applied in practical engineering.

The traditional dam deformation safety monitoring model is established according to mathematics, mechanics, and dam engineering knowledge by confirming the major effect size's affecting factors with the use of regression methods and the evaluation of various affecting factors' parameters. However, dam deformation is uncertain and varied because the high arch dam's working environment is complicated, changeable, and affected by various internal and external factors. The multifactors and multiparts of a dam are connected. The traditional safety monitoring model of the 
deformation of a single measuring point cannot completely reflect the dam's entire deformation status. Moreover, different degrees of underfitting may appear when the traditional dam deformation statistical model is used for regression analysis of safety monitoring information $[6,7]$.

Therefore, a spatial and temporal distribution model construction method of high arch dam is needed to master the changing principles of the high arch dam deformation status. Knowledge of mathematical models must be adopted to construct a high arch deformation safety monitoring model.

\section{High Arch Dam Deformation's Spatial and Temporal Distribution Model}

The traditional high arch dam deformation monitoring model is the single measuring point statistical model that is established according to the single measuring point's data series $[8,9]$. This model cannot completely reflect the high arch dam's comprehensive situation and establish the dam's whole displacement field under a certain time and load combination. Using the measuring points' space coordinates to establish the space displacement field's spatial-temporal distribution model can effectively solve this problem. The mathematical expression is as follows:

$$
\delta=f(H, T, \theta, x, y, z),
$$

where $H$ is the water pressure factor, $T$ is the temperature factor, $\theta$ is the time effect factor, and $x, y, z$ are the space coordinates.

The displacement field generated under the effect of water pressure, temperature load, and time effect factors is the vector field.

$$
\begin{aligned}
\delta(H, T, \theta, x, y, z)= & u(H, T, \theta, x, y, z) i \\
& +v(H, T, \theta, x, y, z) j \\
& +\omega(H, T, \theta, x, y, z) k .
\end{aligned}
$$

The vector field contains three components: $u\left(\delta_{x}\right), v\left(\delta_{y}\right)$, and $\omega\left(\delta_{z}\right), u$ is the displacement along the river (along the upstream and downstream direction), $v$ is the displacement of the vertical direction (along the dam axes direction), and $\omega$ is the displacement that is vertical to the river (Figure 1). Therefore, the abovementioned vector field can be transferred into scalar field for calculation. In accordance with the generality of the mathematical method, $u$ (abbreviated as $\delta$ ) is taken as an example to analyze the main affecting factors when choosing a method.

Formula (1) can be divided into three parts within small deformation ranges and according to deformation components, namely, water pressure component, temperature component, and time effect component.

$$
\begin{aligned}
\delta= & f(H, T, \theta, x, y, z) \\
= & f_{1}(H, x, y, z)+f_{2}(T, x, y, z)+f_{3}(\theta, x, y, z) \\
= & f_{1}[f(H), f(x, y, z)]+f_{2}[f(T), f(x, y, z)] \\
& +f_{3}[f(\theta), f(x, y, z)],
\end{aligned}
$$

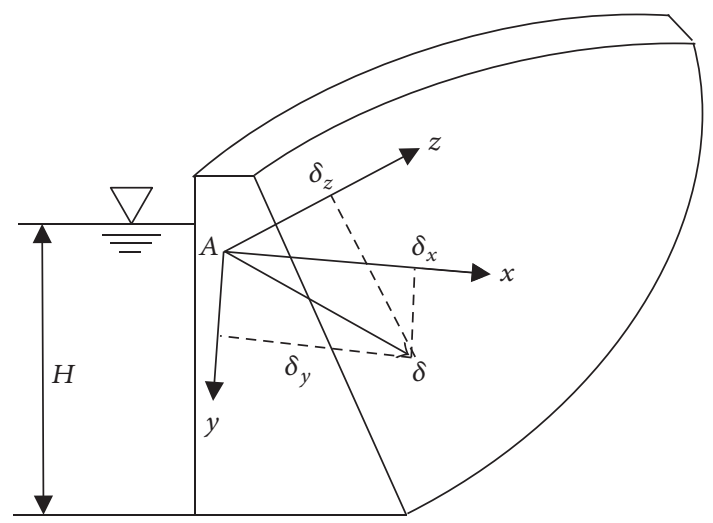

FIgURE 1: Displacement vector and its component's schematic diagram.

where $f(x, y, z)$ is the dam's space deformation displacement that is generated under the effect of a certain load. $f(x, y, z)$ is continuous in the domain $\Omega$ (dam). Therefore, the following can be obtained by using multiple power series and referring to beam curves and the horizontal arch deformation equation:

$$
f(x, y, z)=\sum_{l, m, n=0}^{3} a_{l m n} x^{l} y^{m} z^{n}
$$

where $f(H), f(T)$, and $f(\theta)$ are the water pressure component, temperature component, and time effect component of a fixed point of the dam body or the dam foundation, respectively.

\subsection{Formula of Deformation Component}

2.1.1. Water Pressure Component $f_{1}(H, x, y, z)$. The dam or dam foundation system's whole displacement field under the water load effect is shown as

$$
f_{1}(H, x, y, z)=f_{1}[f(H), f(x, y, z)],
$$

where $f(H)$ is the water pressure of the dam or dam foundation's fixed point. It can be represented by $i$ times the polynomials of water head $H$. The high arch dam takes $i$ as 4 . Thus, the following equation can be obtained:

$$
f(H)=\sum_{i=4}^{4} a_{i} H^{i} .
$$

Formulas (6) and (4) are substituted into formula (5), which can be expanded by using the multiple power series, omitting the higher terms and collecting similar items. The water pressure expression formula of high arch dam deformation spatial and temporal distribution model can be obtained.

$$
\begin{aligned}
f_{1}(H, x, y, z) & =f_{1}[f(H), f(x, y, z)] \\
& =\sum_{k=0}^{4} \sum_{l, m, n=0}^{3} A_{k l m n} H^{k} x^{l} y^{m} z^{n} .
\end{aligned}
$$


2.1.2. Temperature Component $f_{2}(T, x, y, z)$. The dam's space deformation field under the temperature load is as follows:

$$
f_{2}(T, x, y, z)=f_{2}[f(T), f(x, y, z)]
$$

where $f(x, y, z)$ is the dam's displacement field under the effect of a fixed temperature. Formula (4) can be applied.

Temperature factor $f(T)$ is the displacement caused by the temperature load of dam concrete and batholith.

In accordance with the actual measured situation of the temperature, $f(T)$ is divided into two situations.

(1) Concrete Temperature Actual Measurement Information. $f(T)$ can use the concrete actual measured temperature $T_{i}$ as a factor when the concrete thermometer can provide sufficient temperature measurement information. $f(T)$ can use equivalent temperature $\left(\overline{T_{i}}, \beta_{i}\right)$ as a factor when few thermometers are used.

$$
\begin{aligned}
& f(T)=\sum_{i=1}^{m_{1}} b_{i} T_{i}, \\
& f(T)=\sum_{i=1}^{m_{2}} b_{1 i} \overline{T_{i}}+\sum_{i=1}^{m_{2}} b_{2 i} \beta_{i},
\end{aligned}
$$

where $m_{1}$ is the number of concrete thermometers and $b_{i}$ is the regression coefficient. $b_{1 i}$ and $b_{2 i}$ are the regression coefficients. $\bar{T}_{i}$ is the average temperature measured by $i$ layer thermometer. $\beta_{i}$ is the temperature gradient of the $i$ layer thermometer.

(2) No Concrete Temperature, Except Air Temperature and Water Temperature Information, Is Found. The average air temperature and water temperature two days before or the composite type cycles were used as factors.

$$
\begin{aligned}
& f(T)=\sum_{i=1}^{m_{2}} b_{i} T_{i}, \\
& f(T)=\sum_{i=1}^{m_{3}}\left(b_{1 i} \sin \frac{2 \pi i t}{356}+b_{2 i} \cos \frac{2 \pi i t}{356}\right),
\end{aligned}
$$

where $i=1$ is the annual cycle and $i=2$ is a halfyear cycle. $m_{3}$ uses 1 and $2 . b_{1 i}, b_{2 i}$ are the parameters. $t$ is the accumulated days from the monitoring days to the test initiation days.

(3) $f_{2}(T, x, y, z)$ Formula. The different expressions of $f(T)$ show that the above situations can be summarized in formulas (9) and (10). Multiple power series are expanded, higher terms are omitted, and similar items are collected. The final temperature component expression formula can then be obtained.

$$
\begin{gathered}
f_{2}(T, x, y, z)=f_{2}[f(T), f(x, y, z)] \\
=\sum_{j, k=1}^{m_{2}} \sum_{l, m, n=0}^{3} B_{j k l m n} \overline{T_{j}} \beta_{k} x^{l} y^{m} z^{n},
\end{gathered}
$$

$$
\begin{aligned}
f_{2} & (T, x, y, z) \\
& =\sum_{j, k=1}^{1} \sum_{l, m, n=0}^{3} B_{j k l m n} \sin \frac{2 \pi j t}{365} \cos \frac{2 \pi k t}{365} x^{l} y^{m} z^{n} .
\end{aligned}
$$

2.1.3. Time Effect Component $f_{3}(\theta, x, y, z)$. The time effect component can be expressed as

$$
f_{3}(\theta, x, y, z)=f_{3}[f(\theta), f(x, y, z)] .
$$

Time effect factor $f(\theta)$ typically uses the following expression formula:

$$
f(\theta)=c_{1} \theta+c_{2} \ln \theta,
$$

where $\theta$ is calculated from the test start date and increases by 0.01 if one day is added. $c_{1}, c_{2}$ are the regression coefficients.

The final time effect expression formula can be obtained using formula (12).

$$
\begin{aligned}
f_{3}(\theta, x, y, z) & =f_{3}[f(\theta), f(x, y, z)] \\
& =\sum_{j, k=0}^{1} \sum_{l, m, n=0}^{3} C_{j k l m n} \theta_{j} \ln \theta_{k} x^{l} y^{m} z^{n} .
\end{aligned}
$$

2.2. High Arch Dam's Safety Monitoring Statistical Model Based on Spatial and Temporal Distribution. The high arch dam safety monitoring model can be expressed by the following two methods:

$$
\begin{aligned}
& \delta=f(H, T, \theta, x, y, z) \\
& =f_{1}(H, x, y, z)+f_{2}(H, x, y, z) \\
& +f_{3}(H, x, y, z) \text {, } \\
& M_{1}=\sum_{k=0}^{4} \sum_{l, m, n=0}^{3} A_{k l m n} H^{k} x^{l} y^{m} z^{n} \\
& +\sum_{j, k=1}^{m_{2}} \sum_{l, m, n=0}^{3} B_{j k l m n} \overline{T_{j}} \beta_{k} x^{l} y^{m} z^{n} \\
& +\sum_{j, k=0}^{1} \sum_{l, m, n=0}^{3} C_{j k l m n} \theta_{j} \ln \theta_{k} x^{l} y^{m} z^{n}, \\
& \delta=f(H, T, \theta, x, y, z) \\
& =f_{1}(H, x, y, z)+f_{2}(H, x, y, z) \\
& +f_{3}(H, x, y, z), \\
& M_{2}=\sum_{k=0}^{4} \sum_{l, m, n=0}^{3} A_{k l m n} H^{k} x^{l} y^{m} z^{n} \\
& +\sum_{j, k=0}^{1} \sum_{l, m, n=0}^{3} B_{j k l m n} \sin \frac{2 \pi j t}{365} \cos \frac{2 \pi k t}{365} x^{l} y^{m} z^{n} \\
& +\sum_{j, k=0}^{1} \sum_{l, m, n=0}^{3} C_{j k l m n} \theta_{j} \ln \theta_{k} x^{l} y^{m} z^{n},
\end{aligned}
$$




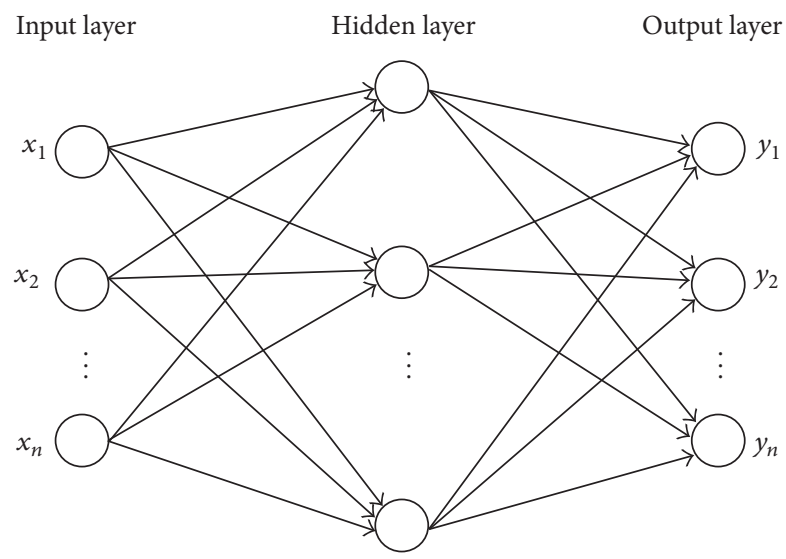

FIGURE 2: BP neural network topology.

where $A_{k l m n}, B_{j k l m n}$, and $C_{j k l m n}$ are fitting coefficients and are calculated by regression calculation based on the dam and batholith's actual measurement information.

\section{High Arch Dam Deformation Safety Monitoring Model Based on Improved BP Neural Network}

3.1. Backpropagation (BP) Neural Network. A BP neural network is a feedforward multilayer neural network based on error backpropagation [10]. It has a strong learning ability and can approach any nonlinear function. It is usually composed of an input layer, a hidden layer, and an output layer (Figure 2).

BP neural network learning occurs according to a given learning function. Nerve cells generate corresponding connection weights to achieve a given mapping relation once the study model is provided. The network minimizes the deviation between the actual weight and the forecast weight. It inversely corrects each layer's weights from the output layer to the middle layers and then returns to the input layer. The process is performed repeatedly until the deviation of the entire network is close to the required minimum value [11].

The BP neural network confirms its structure according to the nonlinear function to be fit. It confirms the input layer nodes according to the number of inputted parameters and the output layer nodes according to the number of outputted parameters. The inputted parameter number in the high arch dam model stress and deformation safety monitoring model is decided by the expression of water pressure, temperature, and time effect factors.

3.2. Artificial Bee Colony $(A B C)$ Algorithm. ABC algorithm is a new group optimization method proposed by Turkish scholar Karaboga, who observed the behavior of bees gathering honey [12]. This method is achieved by the communication, transfer, and cooperation of two types of ABCs. The first type of colony consists of employed bees, which are engaged in finding a food source and then carry and share the information about the food location with other bees. The other colony consists of unemployed bees that are focused on looking for food sources to exploit. The unemployed bees can be either a scout bee, which hunts for food randomly, or an onlooker bee, which searches for the food source by using the information derived from the employed bee [13, 14]. Every searching route in the $\mathrm{ABC}$ algorithm represents a feasible solution of optimized questions. A bee colony searches for the honey source that contains the largest amount of honey through cycle research to obtain the optimal solution [15]. The first step of the ABC algorithm is to generate a randomly initial population $\mathrm{P}$ of $\mathrm{SN}$ solutions $\left(x_{i}(i=1,2, \ldots, \mathrm{SN})\right)$, where SN demonstrates the size of the employed bees or the onlooker bees. Each solution is a $D$-dimension vector, where $D$ is the number of optimization parameters. If the amount of food in a new location is higher than that in the older location, then the bee marks the new location and disregards the old location. The bee colony searches for a new food source around the current food source through iteration [16]. The main steps of the ABC algorithm are as follows:

(1) The leading bee searches the honey source location according to formula (16) and calculates the corresponding honey source fitness value within the neighbor honey source in the memory. To distinguish the new food position from the previous location in its memory, the $\mathrm{ABC}$ algorithm uses the following expression:

$$
v_{i j}=x_{i j}+r_{i j}\left(x_{i j}-x_{k j}\right)
$$

Formula $v_{i j}$ is the nearby honey source location $(i, k \in$ $\{i=1,2, \ldots, \mathrm{SN}\}$ and $i \neq k, j \in\{1,2, \ldots, D\}) . r_{i j}$ is the random number among $[-1,1]$.

(2) A follow bee chooses a food location according to the probability value related to the food source. The formula of the probability is expressed as follows:

$$
P_{i}=\frac{\mathrm{fit}_{i}}{\sum_{i=1}^{\mathrm{SN}} \mathrm{fit}_{i}},
$$

where fit $_{i}$ indicates the fitness of the honey source.

(3) In $\mathrm{ABC}$, the bees locate a food position randomly and persist until another one is found. In $\mathrm{ABC}$, if a food position cannot be further improved by means of a preestablished number of cycles, then that food source is considered to be abandoned. Suppose that the abandoned source is $x_{i}$. Then, the scout bees locate a new food source to be replaced with $x_{i}$. This procedure can be defined as follows:

$$
x_{i}=x_{i, \text { min }}+\operatorname{rand}(0,1)\left(x_{i, \max }-x_{i, \min }\right) .
$$

\subsection{Improved ABC Algorithm}

3.3.1. Improvement of Follow Bee's Honey Source Choosing Probability. In the standard ABC algorithm, a follow bee makes a greedy selection according to the probability regulated in formula (17). However, this selection method 
decreases colony diversity and causes local optimum. Thus, the aim is to change the selection probability according to different research periods. Adaptive proportion is used to achieve this aim [17] and correct the selection probability of formula (17) as follows:

$$
\begin{aligned}
& P_{i}=\frac{\left(\mathrm{fit}_{i}\right)^{\lambda}}{\sum_{i=1}^{\mathrm{SN}}\left(\mathrm{fit}_{i}\right)^{\lambda}}, \\
& \lambda=\frac{k}{\mathrm{fit}_{\text {max }} / \overline{\mathrm{fit}}-1},
\end{aligned}
$$

where fit ${ }_{\max }=\max \left\{\mathrm{fit}_{i}\right\}, i=1,2, \ldots, \mathrm{SN} ; \overline{\mathrm{fit}}=\sum_{i=1}^{\mathrm{SN}} \mathrm{fit}_{i} / \mathrm{SN}$. According to the abovementioned two formulas, the following can be obtained:

(1) When $\overline{\mathrm{fit}} / \mathrm{fit}_{\max } \rightarrow 0, \lambda \rightarrow 0$, fit $_{i}^{\lambda} \rightarrow 1, P_{i}=1 / \mathrm{SN}$, $P_{i}$ is small, and the fitness is bad. Thus, the honey source has living space. The research space is large and ensures diversity.

(2) When $\overline{\text { fit }} /$ fit $_{\max } \rightarrow 1$ and $\lambda \rightarrow \infty$, if fit ${ }_{i}<$ fit $_{\max }$, $P_{i}=0$. If fit $_{i}=$ fit $_{\max }, P_{i}=1 / M ; M$ means the number of individuals that have the highest fitness. The fitness is bad, and the honey source does not have any living space. Thus, the selection can be made only among the honey sources that have high fitness.

The honey source's average fitness is significantly different from the optimal honey source's fitness: (1) $\lambda \rightarrow 0$. This algorithm has high and wide-ranging research ability, and it ensures honey source diversity. (2) $\lambda \rightarrow 0$ changes with the changing of honey source characteristics and seeks balance between wide ranges and elaboration during the selection's middle term. (3) The honey source's average fitness is close to the optimal honey source fitness during the later period of selection. The research space at $\lambda \rightarrow \infty$ is greatly reduced and accelerates the rate of convergence.

3.3.2. Follow Bee's Neighborhood Selection Improvement. The leading bee and follow bee's neighborhood selection method is the same in the standard ABC algorithm. However, the lead bee in the actual honey collection process shares the searched honey source information with a follow bee through a swing dance. The follow bee then chooses a honey source in accordance with a return rate and then selects the honey source within the neighborhood of this food source. Thus, the lead bee and follow bee's neighborhood selection method is different [18]. Therefore, correcting the follow bee's neighborhood search formula and introducing the average Euclidean distance are necessary [19].

$$
m d_{m}=\frac{\sum_{j=1}^{\mathrm{SN}} d(m, j)}{\mathrm{SN}-1} .
$$

Formula $d(m, j)$ shows the Euclidean distance from honey source $x_{m}$ and $x_{j}$. When $d(m, j)<m d_{m}, x_{j}$ is treated as a neighborhood honey source of $x_{m}$.
3.4. Establishing The High Arch Dam Deformation Safety Monitoring Model Based on Improved ABC-BP Neural Network. The BP neural network is combined with the improved $\mathrm{ABC}$ algorithm to establish the dam deformation safety monitoring model. The detailed establishment steps are as follows:

(1) A BP neural network model is established.

(2) The main parameters needed to improve the $A B C$ algorithm are set up. The ABC number is $2 \mathrm{SN}$, among which the lead bee and follow bee quantity is SN. The honey source is updated by multiplying the limit value limit, the largest iteration times MCN, and the honey source dimension. $D=N_{\text {input }} \times N_{\text {hidden }}+$ $N_{\text {hidden }}+N_{\text {hidden }} \times N_{\text {output }}+N_{\text {output }} \cdot N_{\text {input }}, N_{\text {hidden }}$, and $N_{\text {output }}$ are the nerve cell numbers of the BP neural network's input layer, hidden layer, and output layer, respectively.

(3) A small section method is used to generate the initial honey source $x_{i}(i=1,2, \ldots, \mathrm{SN})$. The fitness of each honey source is calculated by the following formula:

$$
\text { fit }_{i}=\frac{1}{E+1} \text {. }
$$

The formula shows the mean squared error of the BP neural network for $i$ solution of $E$.

(4) The lead bee searches for a new honey source according to formula (16). The old honey source is replaced by the new honey source if the new honey source's fitness is better than that of the old honey source. Otherwise, the old honey source's update time is added.

(5) The follow bee calculates the selection probability $P_{i}$ according to adaptive proportion formulas (19) and selects the honey source. It confirms the neighborhood according to average Euclidean distance and $P_{i}$ searches for a new honey source within the neighborhood.

(6) The lead bee turns into a scout bee, searches for a new honey source according to formula (18), and replaces the old source if the honey source's update time exceeds the preset time limit. It chooses the honey source that has the best fitness.

(7) The optimal solution to weights and thresholds of BP neural network is added if the iteration times of the $\mathrm{ABC}$ algorithm reach MCN. Otherwise, step (4) is followed.

(8) The sample number is inputted, and dam deformation monitoring and forecasting are implemented.

\section{Case Study}

4.1. Project Profile. The dam is located in the Yi Autonomous Prefecture, Liangshan, Sichuan. The dam is a controlled cascade reservoir located in the Ya-lung River's middle and downstream areas, and it was constructed according to a 
hydropower development plan. The project is mainly used to generate electricity and has an annual adjusted capacity. It has a great compensation and regulation function on the downstream cascade. The project is of type 1 and is considered a first-class project. Its permanent hydraulic structure belongs to the first-class structure. The reservoir's normal pool level is $1880 \mathrm{~m}$, and the dead water level is $1800 \mathrm{~m}$. Its reservoir capacity under normal pool level is 7.76 billion $\mathrm{m}^{3}$.

The dam is a concrete double-curvature arch dam. The dam crest height is $1885 \mathrm{~m}$. The minimum height of the dam's foundation is $1580 \mathrm{~m}$. The maximum dam height is $306 \mathrm{~m}$. The dam crest's width is $16 \mathrm{~m}$, and the dam foundation's thickness is $63 \mathrm{~m}$. The thickness and height ratio is 0.207 . The project achieves river closure on December 4, 2016, and has achieved the four stages of water reservoir since November 30, 2012.

The dam's horizontal displacement monitoring is performed through a V-perpendicular line and a reversed pendulum. Approximately 57 measuring points are set up at the 1\#, 5\#, 9\#, 11\#, 13\#, 16\#, 19\#, and 23\# dam sections of the mountain body at the left bank and the dam crest's elevations of $1885,1829,1778,1730,1664$, and $1601 \mathrm{~m}$ of the mountain body at the right bank.

4.2. Application Example. The PL13-3 measuring points' monitoring data from the $13 \#$ dam section and the 585 values measured from January 2, 2013, to August 10, 2014, are used in this study. The traditional statistical model, BP neural network, and the improved ABC-BP neural network are then used to establish the structural safety monitoring model of high arch dam.

4.2.1. Statistical Model. According to the PL13-3 measuring points' data, the expression $(f(\theta, x, y, z)=$ $\left.\sum_{l, m, n=0}^{3} a_{l m n} x^{l} y^{m} z^{n}\right)$ in the temporal-spatial distribution model is set to a constant because $x, y, z$ of the fixed measuring points are definite. Then, the statistical model of a single measuring point's deformation displacement can be constructed as follows by collating the high arch dam's safety monitoring statistical model based on its spatial and temporal distribution:

$$
\begin{aligned}
\delta= & f(H, T, \theta, x, y, z) \\
= & f_{1}(H, x, y, z)+f_{2}(T, x, y, z)+f_{3}(\theta, x, y, z) \\
= & f_{1}[f(H), f(x, y, z)]+f_{2}[f(T), f(x, y, z)] \\
& +f_{3}[f(\theta), f(x, y, z)]=f(H)+f(T)+f(\theta) \\
= & a_{0}+\sum_{i=1}^{4} a_{i} H^{i}+\sum_{i=1}^{2}\left(b_{1 i} \sin \frac{2 \pi i t}{365}+b_{2 i} \cos \frac{2 \pi i t}{365}\right) \\
& +c_{1} \theta+c_{2} \ln \theta
\end{aligned}
$$

where $a_{0}, a_{i}, b_{1 i}, b_{2 i}, c_{1}$, and $c_{2}$ are fitting coefficients.

Then, the regression calculation is performed based on the displacement monitoring data of PL13-3, and every measuring point's fitting coefficient is shown in Table 1.

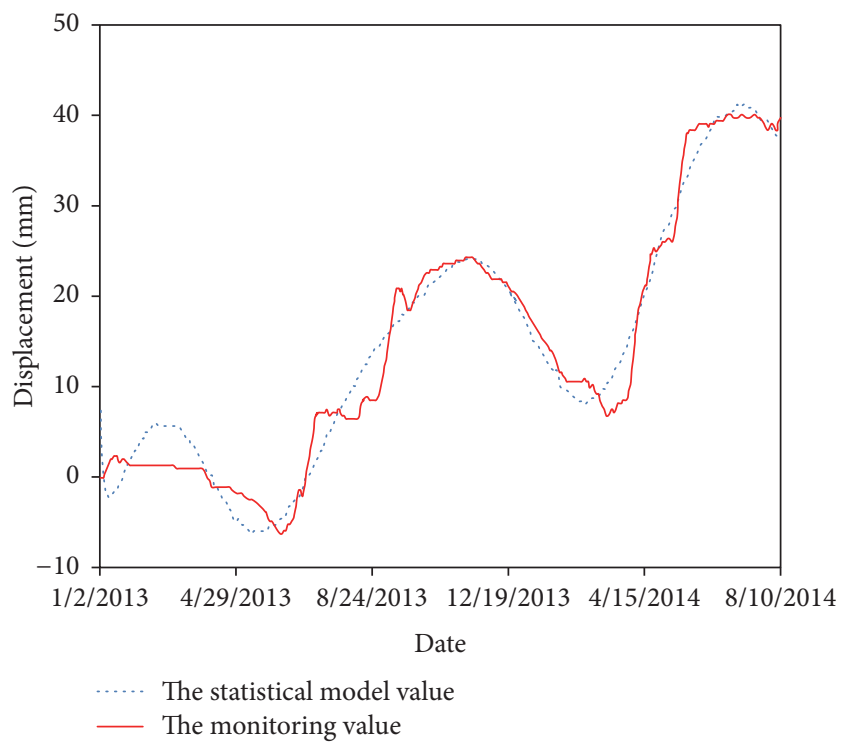

FIGURE 3: Statistical model's fitting process line.

The statistical model's fitting process line is shown in Figure 3.

4.2.2. BP and Improved ABC-BP Model. The high arch dam deformation is affected by time effect, temperature, and water pressure. Eight affecting factors are chosen, and BP and ABCBP neural network models are established. Two temperature factors, $\sin (2 \pi t / 365)$ and $\cos (2 \pi t / 365)$, are chosen. Two time effect factors, $\theta$ and $\ln \theta$, are chosen. $\theta$ is time. Four water pressure factors, $H, H^{2}, H^{3}$, and $H^{4}$, are chosen. Therefore, a total of eight nodes are present in the BP neural network's input layer, and the output layer's value is measured.

The BP neural network model's hidden layer adopts five nodes to establish the model. The ABC-BP and BP models were trained 1000 times. The BP model uses the BP algorithm only and trains the neural network 1000 times. With regard to the improved ABC-BP model, the leading bee and follow bee are each set to 100 , respectively, and the limit is set to 300 . The model first performs the ABC iteration and then uses the BP algorithm to train the neural network. To choose the proper number of iterations, we can compare and select the fitting precision of the mean square deviation $F_{\text {MSE }}$ given by formula (23). The result is shown in Table 2.

$$
F_{\mathrm{MSE}}=\frac{1}{n} \sqrt{\sum_{i=1}^{n}\left(y_{i}-\hat{y}_{i}\right)^{2}},
$$

where $y_{i}$ shows the number $i$ monitoring value and $\widehat{y}_{i}$ shows the number $i$ model calculation value.

The results in Table 2 show that the improved ABC-BP model first performs $300 \mathrm{ABC}$ iterations and then uses the $\mathrm{BP}$ algorithm to train the neural network 700 times, thereby indicating that this model is the best choice. Therefore, we chose this method to construct the model.

To achieve better fitting and forecasting, the comparison and selection results of Table 2 are applied, and formula (23) 
TABLE 1: Fitting coefficient of the statistic model.

\begin{tabular}{|c|c|c|c|c|c|c|c|c|c|c|}
\hline Constant term & & Water $\mathrm{p}$ & ssure factors & & & Temper & ure factors & & Time e & t factors \\
\hline $\mathrm{a} 0$ & al & a2 & a3 & $\mathrm{a} 4$ & b11 & b12 & b21 & b22 & $\mathrm{cl}$ & c2 \\
\hline-5.5877 & 0.4869 & $-3.57 E-03$ & $1.17 E-05$ & $-8.08 E-09$ & 3.1721 & 0.7244 & -0.1338 & -0.155 & -0.3248 & -1.3907 \\
\hline
\end{tabular}

TABLE 2: Comparison and selection of the improved ABC-BP model's iteration number.

\begin{tabular}{|c|c|c|c|c|}
\hline & $\begin{array}{c}\text { ABC: } 200 \\
\text { BP: } 800\end{array}$ & $\begin{array}{c}\text { ABC: } 250 \\
\text { BP: } 750\end{array}$ & $\begin{array}{c}\text { ABC: } 300 \\
\text { BP: } 700\end{array}$ & $\begin{array}{c}\text { ABC: } 350 \\
\text { BP: } 650\end{array}$ \\
\hline$F_{\mathrm{MSE}}$ & 0.09021 & 0.08893 & 0.07916 & 0.08672 \\
\hline
\end{tabular}

TABLE 3: Comparison and selection between different data sets.

\begin{tabular}{|c|c|c|c|c|c|}
\hline & $\begin{array}{c}N=500 \\
M=30\end{array}$ & $\begin{array}{c}N=540 \\
M=30\end{array}$ & $\begin{array}{c}N=540 \\
M=35\end{array}$ & $\begin{array}{c}N=540 \\
M=40\end{array}$ & $\begin{array}{c}N=540 \\
M=45\end{array}$ \\
\hline$F_{\mathrm{MSE}}$ & 0.08277 & 0.08091 & 0.07957 & 0.07949 & 0.08128 \\
\hline
\end{tabular}

is used to analyze the fitting and the forecasting precision of the different data sets (the first $N$ groups are used to fit, and the last $M$ groups are used to forecast). The result is shown in Table 3.

The results in Table 3 show that the precision of the third and fourth groups is better than that of the other three groups. Moreover, the precision of the third group is similar to that of the fourth group. However, as the data volume of group three is less than that of group four, the calculation speed of group three will be faster than that of group four. Thus, the first 540 groups of data are used for fitting, and the latter 35 groups of data are used for forecasting. Finally, the BP and the improved ABC-BP model can be constructed.

The BP neural network calculation result is shown in Figure 4. The improved ABC-BP neural network calculation result is shown in Figure 5.

This study separately uses the improved ABC-BP neural network and traditional BP neural network to establish 50 groups of forecasting models on the high arch dam's horizontal displacement information and further improve the $\mathrm{ABC}-\mathrm{BP}$ neural network's advantage in data monitoring and forecasting. This study summarizes the first 540 fitting stage data and the latter 35 forecast stage data and calculates the standard deviation between 50 times of fitting stage data and the forecast stage data. The standard deviation of the two stages is summarized, and a comparison diagram of the three models' standard deviations of these two stages is drawn (Figure 6).

The following findings were obtained:

(1) The BP neural network and the improved ABCBP neural network have a better fitting effect on dam deformation data monitoring compared with traditional models. The improved ABC-BP model also has certain advantages in data fitting over the $\mathrm{BP}$ neural network model.

(2) Most of the standard deviations of the ABC-BP neural network in the forecast stage are less than the traditional BP neural network's standard deviation.

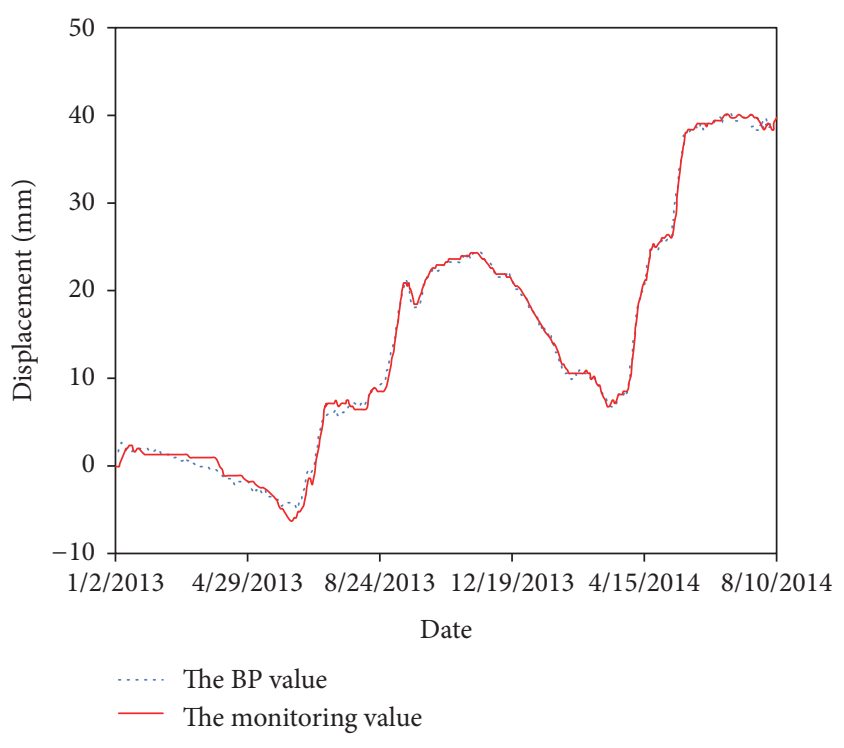

FIGURE 4: BP neural network model's fitting process line.

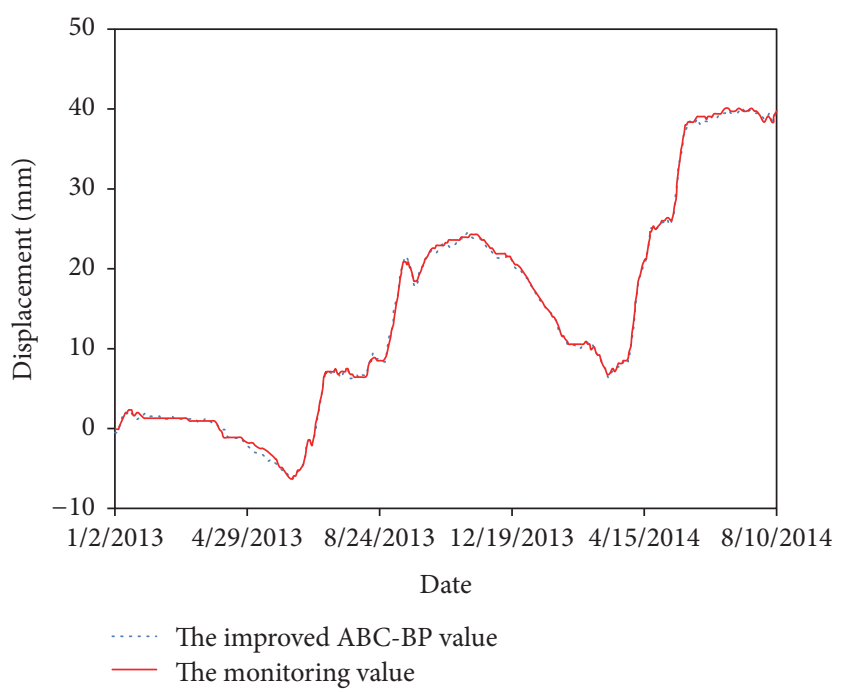

FIGURE 5: Improved ABC-BP neural network's fitting process line.

Thus, the improved ABC-BP neural network in the forecast stage has better advantages than the $\mathrm{BP}$ neural network.

\section{Conclusion}

This study discusses the factor selection and model optimization method of a high arch dam deformation safety monitoring model on the basis of research on the traditional 


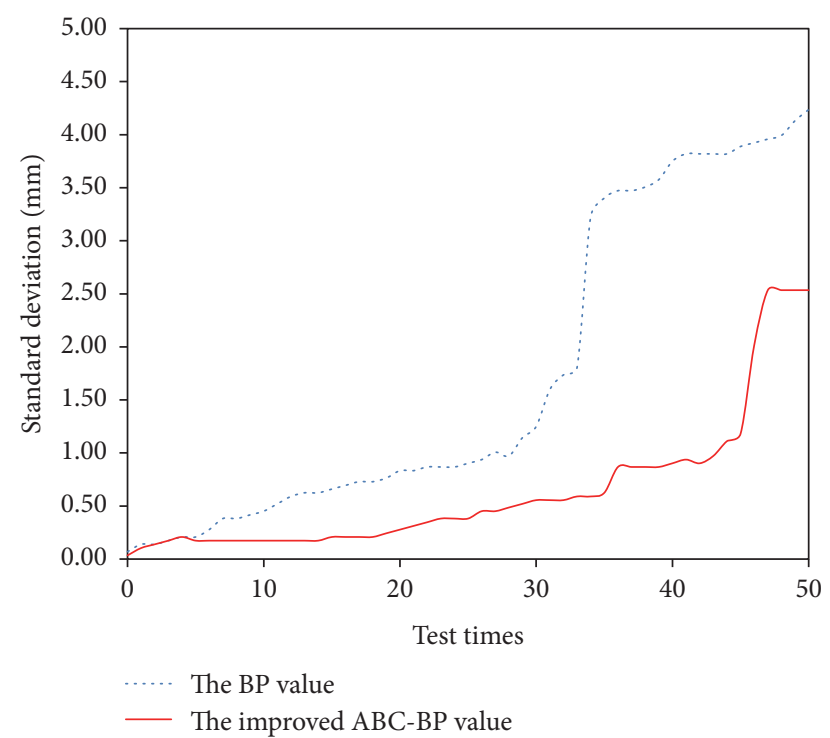

FIGURE 6: Comparison between the forecast stage's standard deviation of the BP neural network and the improved ABC-BP neural network.

safety monitoring statistical model's establishment theory. The following achievements were made:

(1) The study focuses on the high arch dam deformation's spatial and temporal distribution principles and the safety monitoring model's establishment methods. It emphasizes and establishes the high arch dam deformation's safety monitoring spatial and temporal model. Moreover, it discusses the establishment of a suitable high arch deformation safety monitoring model according to the water pressure, temperature, and time effect components.

(2) The method uses few control parameters, has a simple operation, and exhibits improved optimal performance characteristics of the $\mathrm{ABC}$ algorithm to optimize the initial weight value and threshold of the BP neural network. It adopts the adaptive proportion to improve the follow bee's food source selection probability and uses the average Euclidean distance to improve the follow bee's neighborhood selection. It effectively overcomes the standard $\mathrm{ABC}$ algorithm's local optimum shortcoming and has more accurate fitting and forecasting ability. Thus, the method is suitable for dam deformation monitoring.

(3) This research establishes a high arch dam deformation safety monitoring model based on the improved $\mathrm{ABC}-\mathrm{BP}$ model. The method can effectively improve the model's fitting and forecasting ability for an engineering project. This method can provide a reference for the establishment of a dam safety monitoring model and for the creation of a forecasting model in other fields.

\section{Competing Interests}

The authors declare that there is no conflict of interests regarding the publication of this paper.

\section{Acknowledgments}

This work was supported by National Natural Science Foundation of China (Grant nos. 51139001, 41323001, 51479054, 51579086, 51379068, 51579083, 51279052, and 51579085), National Key Research and Development Project (2016YFC0401601), Jiangsu Natural Science Foundation (Grant no. BK20140039), Research Fund for the Doctoral Program of Higher Education of China (Grant no. 20130094110010), a project funded by the Priority Academic Program Development of Jiangsu Higher Education Institutions (Grant no. YS11001), Jiangsu Province "Six Talent Peaks" Project (Grant nos. JY-008 and JY-003), and Central University Basic Research Project (Grant no. 2015B20714).

\section{References}

[1] J. M. W. Brownjohn, "Structural health monitoring of civil infrastructure," Philosophical Transactions of the Royal Society A: Mathematical, Physical and Engineering Sciences, vol. 365, no. 1851, pp. 589-622, 2007.

[2] A. De Sortis and P. Paoliani, "Statistical analysis and structural identification in concrete dam monitoring," Engineering Structures, vol. 29, no. 1, pp. 110-120, 2007.

[3] J. Chen, "Using measured displacement data to estimate the actual modulus of elasticity of the dam concrete," Hydropower and Pumped Storage, vol. 2, pp. 3-9, 1983.

[4] $\mathrm{Z}$. Wu, "The deterministic model and mixed model of safety monitoring for concrete dams," Journal of Hydraulic Engineering, vol. 5, pp. 64-70, 1989.

[5] C. Gu, D. Qin, Z. Li, and X. Zheng, "Study on semi-parametric statistical model of safety monitoring of cracks in concrete dams," Mathematical Problems in Engineering, vol. 2013, Article ID 874629, 9 pages, 2013.

[6] H. Su, Z. Chen, and Z. Wen, "Performance improvement method of support vector machine-based model monitoring dam safety," Structural Control and Health Monitoring, vol. 23, no. 2, pp. 252-266, 2016.

[7] H. Huang, B. Chen, and C. Liu, "Safety monitoring of a super-high dam using optimal kernel partial least squares," Mathematical Problems in Engineering, vol. 2015, Article ID 571594, 13 pages, 2015.

[8] J. Mata, A. Tavares De Castro, and J. Sá Da Costa, "Constructing statistical models for arch dam deformation," Structural Control and Health Monitoring, vol. 21, no. 3, pp. 423-427, 2014.

[9] C. Gu and Z. Wu, Safety Monitoring of dams and Dam Foundations. Theories \& Methods and Their Application, Hohai University Press, Nanjing, China, 2006.

[10] I. A. Basheer and M. Hajmeer, "Artificial neural networks: fundamentals, computing, design, and application," Journal of Microbiological Methods, vol. 43, no. 1, pp. 3-31, 2000.

[11] X. Wu, H. Shi, and Z. Li, "Method of groundwater level prediction in Coal Mine Based on BP neural network," Industry and Mine Automation, vol. 5, pp. 23-21, 2006. 
[12] D. Karaboga and B. Akay, "A comparative study of artificial Bee colony algorithm," Applied Mathematics and Computation, vol. 214, no. 1, pp. 108-132, 2009.

[13] D. Karaboga and C. Ozturk, "A novel clustering approach: Artificial Bee Colony (ABC) algorithm," Applied Soft Computing, vol. 11, no. 1, pp. 652-657, 2011.

[14] D. Karaboga and B. Basturk, "A powerful and efficient algorithm for numerical function optimization: artificial bee colony (ABC) algorithm," Journal of Global Optimization, vol. 39, no. 3, pp. 459-471, 2007.

[15] L. Xu, M. Wu, and Z. Yuan, "Improved artificial bee colony algorithm for UAV path planning research," Fire Control \& Command Control, vol. 40, no. 1, pp. 62-66, 2015.

[16] C. Chen, C. Shao, W. Wei et al., "ABC BP model in the concrete double curvature arch dam deformation monitoring applications," Water Resources an Power, vol. 31, no. 8, pp. 112114, 2013.

[17] X. Yang and C. Liu, "Adaptive proportional selection strategy in genetic algorithm," Computer Engineering and Applications, vol. 43, no. 20, pp. 27-25, 2007.

[18] D. Karaboga and B. Gorkemli, "A quick artificial bee colony (qABC) algorithm and its performance on optimization problems," Applied Soft Computing, vol. 23, pp. 227-238, 2014.

[19] W. Li and X. Nan, "Based on improved ABC LSSVM redox potential prediction study," Computer Measurement \& Control, vol. 22, no. 12, pp. 3915-3918, 2014. 


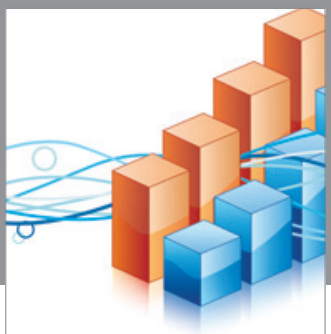

Advances in

Operations Research

vatem alat4

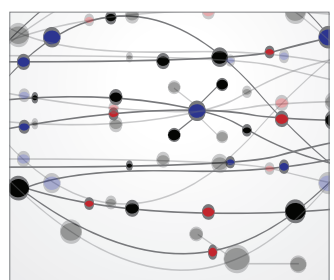

\section{The Scientific} World Journal
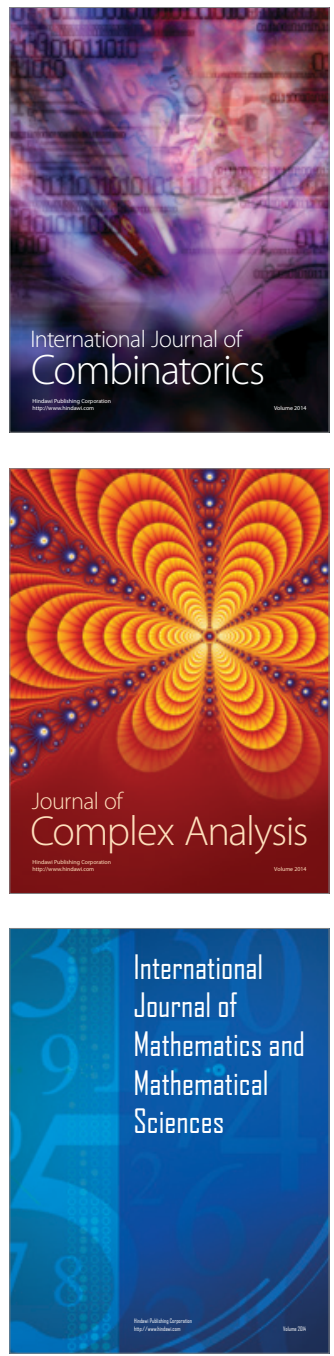
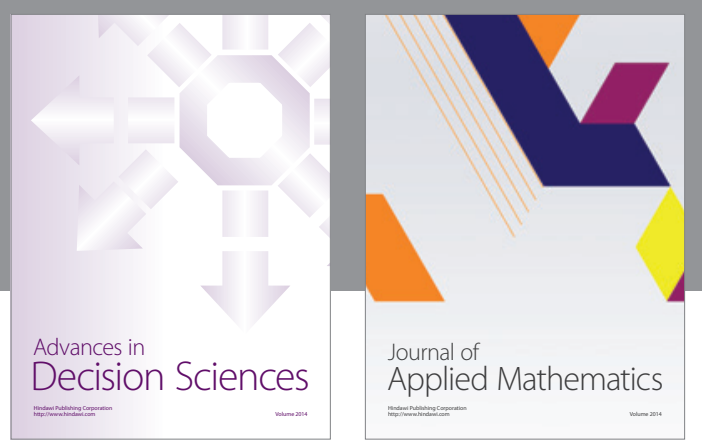

Algebra

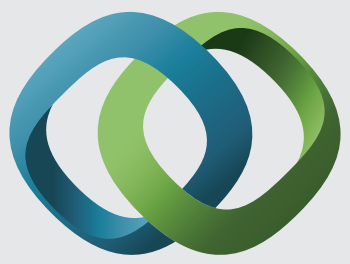

\section{Hindawi}

Submit your manuscripts at

http://www.hindawi.com
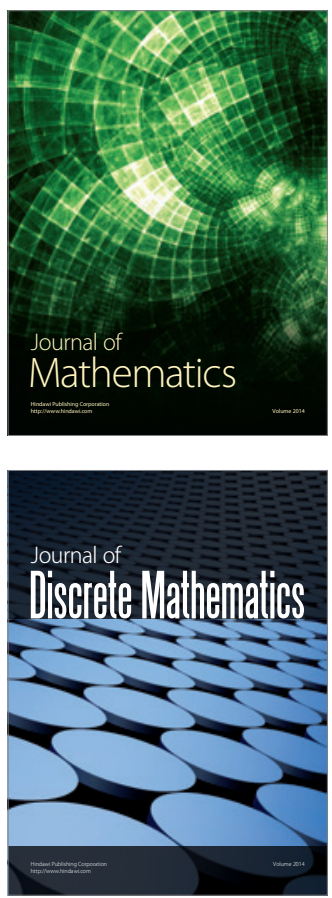

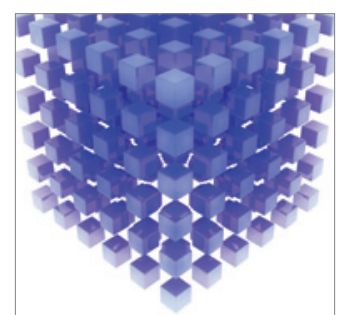

Mathematical Problems in Engineering
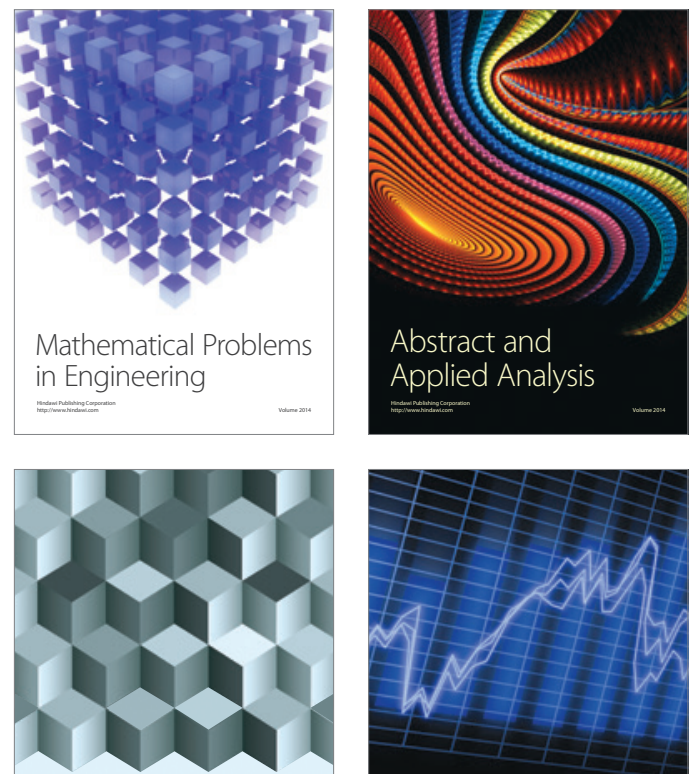

Journal of

Function Spaces

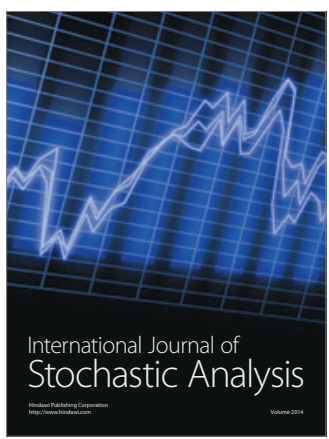

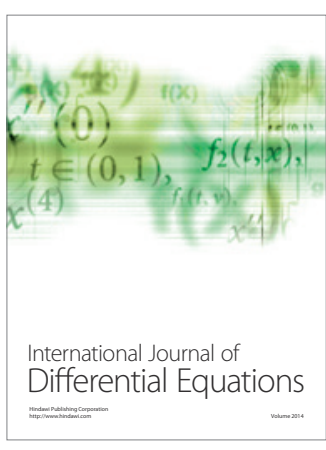
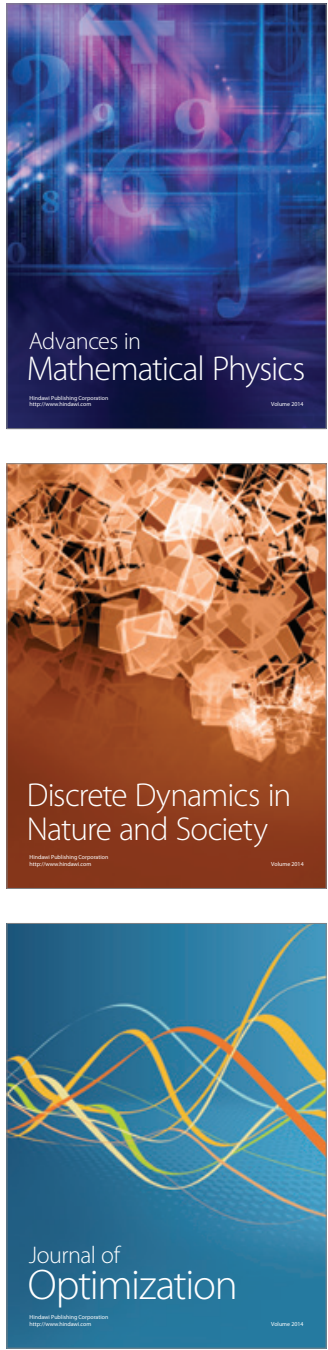